\title{
Evaluación de un módulo de docencia ambulatoria de enfermedades respiratorias en el currículo de pregrado de Medicina
}

\author{
ISABEL LEIVA R. ${ }^{1}$, MARCELA BITRAN C. ${ }^{2}$, FERNANDO SALDÍAS P. ${ }^{1}$
}

${ }^{1}$ Departamento

de Enfermedades Respiratorias.

${ }^{2}$ Centro de Educación Médica.

Facultad de Medicina,

Pontificia Universidad Católica de Chile, Santiago,

Recibido el 29 de agosto de 2011, aceptado el 6 de enero de 2012.

Correspondencia a: Dra. Isabel Leiva Rodríguez Departamento de Enfermedades Respiratorias Pontificia Universidad Católica de Chile. Marcoleta 350, Santiago, Chile. Teléfonos: (562) 6331541 (562) 3543242 Fax: (562) 6335255

E-mail: ileiva@med.puc.cl

\section{Evaluation of a teaching ambulatory module of respiratory diseases in the undergraduate medical curriculum}

Background: As the focus of healthcare provision shifts towards ambulatory care, increasing attention must now be given to develop opportunities for clinical teaching in this setting. Aim: To assess teacher and students' views about the strengths and weaknesses of real and simulated patient interactions for teaching undergraduate students clinical skills in the ambulatory setting. Material and Methods: Fourthyear medical students were exposed in a systematic way, during two weeks, to real and simulated patients in an outpatient clinic, who presented common respiratory problems, such as asthma, chronic obstructive pulmonary disease, smoking and sleep apnea syndrome. After the clinical interview, students received feedback from the tutor and their peers. The module was assessed interviewing the teachers and evaluating the results qualitatively. Students evaluated the contents and quality of teaching at the end of the rotation. Results: Tutors identified the factors that facilitate ambulatory teaching. These depended on the module design, resources and patient care, of characteristics of students and their participation, leadership and interaction with professors. They also identified factors that hamper teaching activities such as availability of resources, student motivation and academic recognition. Most students evaluated favorably the interaction with real and simulated patients in the ambulatory setting. Conclusions: Teaching in the ambulatory setting was well evaluated by students and teachers. The use of qualitative methodology allowed contrasting the opinions of teachers and students.

(Rev Med Chile 2012; 140: 616-624).

Key words: Ambulatory care; Education, medical; Primary health care; Students, medical.
E n la última década hemos sido testigos de un cambio dramático en la organización de los sistemas de atención de salud de la población a nivel mundial, derivándose la atención de los pacientes desde el ambiente hospitalario hacia el ámbito ambulatorio․ Es así como en la práctica clínica actual, en el hospital se concentra la atención de los pacientes más graves y com- plejos, que requieren mayores recursos para su evaluación y tratamiento, y que son cada vez más representativos de las subespecialidades médicas y menos representativos de la práctica médica cotidiana $a^{2-5}$. La necesidad actual de contención de los costos de salud y las expectativas de los pacientes han llevado a acortar las estadías de los pacientes en el hospital, favoreciendo el manejo 
ambulatorio de la mayor parte de los problemas de salud de la población ${ }^{6-8}$.

Consistente con estos cambios en la atención de salud, la formación clínica de los estudiantes de medicina ha presentado un viraje de la educación médica de pregrado hacia el ámbito ambulatorio $^{9-12}$. En la actualidad, la educación médica de pregrado tradicional, centrada en la atención de pacientes hospitalizados, es considerada menos favorable para el aprendizaje de las competencias clínicas de los estudiantesy el ámbito ambulatorio ofrecería mayores ventajas- ${ }^{4-7,13,14}$.

Similar a lo acontecido en los centros de formación médica del hemisferio norte, en la Escuela de Medicina de la Pontificia Universidad Católica de Chile, los cambios curriculares de la carrera han determinado un incremento significativo de la docencia ambulatoria ${ }^{15-17}$. En el ciclo preclínico y clínico de la carrera, los alumnos aprenden entrevistando a "pacientes entrenados" o actores en salas-espejo y atendiendo a pacientes en centros de atención ambulatoria, empleando estrategias educacionales modernas como enseñanza centrada en el estudiante, aprendizaje basado en casos clínicos y seminarios de grupos pequeños ${ }^{15-17}$. A partir del 2004 se implementaron los módulos de docencia ambulatoria en salas-espejo para estudiantes de cuarto año de medicina en el Centro Médico San Joaquín, los cuales han facilitado el aprendizaje de destrezas clínicas y habilidades comunicacionales en el contexto de distintas subespecialidades médicas. El propósito de este estudio es examinar las fortalezas y debilidades del programa de docencia ambulatoria de enfermedades respiratorias de cuarto año de medicina en nuestra institución desde el punto de vista de los docentes y de los estudiantes.

\section{Sujetos y Métodos}

\section{Descripción del módulo docente}

El módulo ambulatorio de enfermedades respiratorias forma parte del curso teórico-práctico de cuarto año de medicina, que incluye actividades prácticas durante la mañana y teóricas durante la tarde. El objetivo de este módulo es promover las habilidades comunicacionales y el aprendizaje de destrezas clínicas básicas relacionadas con el diagnóstico y manejo de enfermedades respiratorias crónicas prevalentes en la comunidad ${ }^{18-20}$, tales como la enfermedad pulmonar obstructiva crónica (EPOC), asma bronquial, síndrome de apneas obstructivas del sueño (SAOS) y tabaquismo; poniendo énfasis en la entrevista clínica, examen físico y atención centrada en el paciente.

La metodología educativa está basada en la docencia tutorial de grupos pequeños (5-7 estudiantes), integrando el aprendizaje teórico-práctico de la especialidad mediante actividades estructuradas (Tabla 1). Las actividades se realizan en una sala que cuenta con espejo unidireccional, sistema audiovisual, acceso a computadores, exámenes radiográficos y de función pulmonar y material bibliográfico de referencia. Los pacientes atendidos han sido seleccionados de acuerdo a la patología y a su disposición a colaborar con la actividad docente. Los pacientes simulados son actores entrenados que pertenecen a la Unidad de Pacientes

Tabla 1. Descripción de las actividades docentes realizadas en el módulo ambulatorio de enfermedades respiratorias del adulto

\section{Actividades teóricas}

a) Lecturas programadas de asma bronquial, enfermedad pulmonar obstructiva crónica, tabaquismo y síndrome de apneas obstructivas del sueño

b) Módulos de autoinstrucción de enfermedades respiratorias del adulto

c) Sesiones teóricas y discusión de casos clínicos

\section{Actividades prácticas}

a) Entrevista clínica a pacientes reales (asma y EPOC) y paciente simulado (tabaquismo y SAOS)

b) Taller práctico de laboratorio de función pulmonar: Espirometría, prueba de provocación bronquial con metacolina, pruebas cutáneas de alergia, prueba de caminata en 6 minutos, medición de la presión inspiratoria máxima

c) Taller práctico de terapia inhalatoria y uso de inhaladores

\section{Actividades de evaluación}

a) Sesiones de retroalimentación dirigidas por el docente (feedback)

b) Evaluación del estudiante mediante pauta ad hoc de la Escuela de Medicina y evaluación clínica objetiva estructurada (ECOE)

c) Evaluación de la docencia tutorial y del módulo ambulatorio realizada por los estudiantes mediante encuestas elaboradas por el Centro de Educación Médica 
Entrenados de la Escuela de Medicina, quienes han sido preparados con una pauta estructurada que permite estandarizar la entrevista clínica y la interacción con los estudiantes.

\section{Evaluación de la actividad docente}

Durante el período 2004-2010, se han aplicado metodologías cuantitativas para evaluar el cumplimiento de los objetivos de aprendizaje y el desempeño de los docentes en el módulo ambulatorio por parte de los estudiantes. En el primer semestre de 2011 se agregó metodología cualitativa ${ }^{21,22}$ para el levantamiento de la información relevante en relación a la evaluación del módulo por parte de los docentes.

\section{Metodología cuantitativa}

Al finalizar la rotación por la sala espejo, los estudiantes respondieron dos cuestionarios dirigidos a evaluar la calidad de la docencia tutorial y los contenidos específicos del módulo ${ }^{23}$.

\section{Metodología cualitativa}

Se realizaron entrevistas semi-estructuradas a seis docentes que participaron en la actividad ambulatoria, para evaluar las fortalezas y debilidades del programa, se exploraron áreas previamente definidas (Tabla 2). Los docentes firmaron un consentimiento informado antes de la entrevista; en dicho documento se explicitaron los objetivos de la investigación y los resguardos éticos considerados para proteger la confidencialidad del entrevistado. Las entrevistas fueron grabadas y transcritas textualmente y los textos fueron analizados de acuerdo al modelo sugerido por la Teoría Fundada ${ }^{21}$, con el propósito de generar conceptos

Tabla 2. Ejes temáticos de la entrevista semiestructurada realizada a los docentes del módulo ambulatorio de enfermedades respiratorias

1. Fortalezas y debilidades de la docencia ambulatoria de enfermedades respiratorias

2. Aspectos específicos de la docencia tutorial y de la dinámica de grupo

3. Diferencias entre la docencia de aspectos teóricos y aspectos prácticos de la actividad clínica y desarrollo de habilidades comunicacionales

4. Uso de los recursos docentes y categorías respecto a las percepciones de los participantes. Para asegurar la confiabilidad de los resultados, tres revisores analizaron y codificaron en forma independiente las entrevistas y se empleó la estrategia de triangulación de los interpretantes ${ }^{22}$ de manera que las categorías generadas fueran validadas a través de un acuerdo intersubjetivo. Asimismo, la generación de categorías y subcategorías conceptuales se orientaron por el criterio de descripción densa, procurando comprender e interpretar las respuestas de los entrevistados en forma contextualizada, de manera de describir plausiblemente sus experiencias.

\section{Análisis estadístico}

Los resultados de las encuestas semiestructuradas realizadas a los estudiantes (evaluación cuantitativa) fueron expresados como valores promedio y los diferentes ítems de las encuestas fueron comparados mediante análisis de varianza considerando significativo un valor de $\mathrm{p}<0,05$.

\section{Resultados}

\section{Evaluación de los docentes}

La evaluación del módulo ambulatorio de enfermedades respiratorias realizada por los docentes permitió identificar los factores que facilitan la docencia dependiente del módulo de aprendizaje (Tabla 3), los estudiantes (Tabla 4) y los docentes (Tabla 5); y por otra parte, los factores que dificultan la docencia (Tabla 6).

\section{Facilitadores de la docencia dependientes del módulo de aprendizaje}

Las condiciones que favorecen la docencia ambulatoria dependientes de la estructura y diseño del módulo de aprendizaje fueron agrupadas en aquellas dependientes de los recursos, del diseño, de las características de la docencia con atención de pacientes y de la docencia ambulatoria en general.

Respecto a los recursos, el más relevante fue la disponibilidad de pacientes y su disposición a colaborar con la docencia. Esto lo expresan los docentes de la siguiente forma:

"Los pacientes para mi es lo más importante, es la razón de ser de la docencia ambulatoria", "vamos a ver a un paciente, esta es la vida real y ahí los sitúo en su ambiente", "yo les aseguro que se van a enfrentar a mil pacientes como los que vamos a ver". 
Módulo de docencia ambulatoria de enfermedades respiratorias - I. Leiva et al

Tabla 3. Condiciones que favorecen la docencia ambulatoria dependientes de la estructura y diseño del módulo de enfermedades respiratorias del adulto

\begin{tabular}{|c|c|}
\hline \multicolumn{2}{|c|}{ Recursos } \\
\hline a) & $\begin{array}{l}\text { Los pacientes reales (seleccionados, permiten expe- } \\
\text { riencias repetidas) }\end{array}$ \\
\hline b) & Los pacientes simulados (actores entrenados) \\
\hline c) & Los docentes de la especialidad \\
\hline d) & Consulta médica y sala espejo \\
\hline e) & Sistema audiovisual y computacional \\
\hline f) & Laboratorio de función pulmonar \\
\hline \multicolumn{2}{|r|}{ Diseño de la docencia ambulatoria } \\
\hline a) & Dirigida a las necesidades del estudiante \\
\hline b) & $\begin{array}{l}\text { Actividad docente organizada, estructurada y estan- } \\
\text { darizada }\end{array}$ \\
\hline c) & Objetivos precisos, factibles de cumplir \\
\hline d) & $\begin{array}{l}\text { Favorece el desarrollo de habilidades clínicas generales, } \\
\text { de la especialidad y transversales (éticas, comunicacio- } \\
\text { nales, profesionalismo) }\end{array}$ \\
\hline e) & $\begin{array}{l}\text { Ambiente grato, controlado, seguro, favorece el } \\
\text { aprendizaje }\end{array}$ \\
\hline f) & $\begin{array}{l}\text { Dedicación exclusiva a esta actividad, se libera al do- } \\
\text { cente de otras obligaciones }\end{array}$ \\
\hline \multicolumn{2}{|r|}{$\begin{array}{l}\text { Características de la docencia con atención de } \\
\text { pacientes }\end{array}$} \\
\hline a) & Favorece el modelaje del docente (sirve de modelo) \\
\hline b) & $\begin{array}{l}\text { Permite enseñar en forma activa, resolviendo pro- } \\
\text { blemas }\end{array}$ \\
\hline c) & Permite entregar feedback inmediato \\
\hline d) & Refuerza el conocimiento teórico \\
\hline e) & Múltiples oportunidades de aprendizaje \\
\hline f) & Promueve aprendizajes significativos \\
\hline g) & $\begin{array}{l}\text { Sistema ganar-ganar: Docentes, estudiantes, pacientes } \\
\text { de escasos recursos }\end{array}$ \\
\hline h) & Permite presenciar cambios en los estudiantes \\
\hline i) & $\begin{array}{l}\text { Permite evaluar a los pacientes en forma integral en } \\
\text { tiempo limitado }\end{array}$ \\
\hline j) & Integra a los familiares en la atención del paciente \\
\hline \multicolumn{2}{|r|}{ De la docencia ambulatoria en general } \\
\hline a) & Es la modalidad más frecuente de atención de salud \\
\hline b) & Los pacientes son representativos de la realidad \\
\hline c) & Oferta variada de patologías \\
\hline d) & Posibilidad de conocer la evolución del paciente \\
\hline e) & Sistema eficiente, optimiza el tiempo \\
\hline f) & No se tiene la presión de decisiones vida-muerte \\
\hline g) & La supervisión docente directa es constante \\
\hline h) & Complementaria a la atención hospitalaria \\
\hline
\end{tabular}

Tabla 4. Condiciones que favorecen la docencia en el módulo ambulatorio de enfermedades respiratorias del adulto dependientes de los estudiantes

\section{Propias de los estudiantes}

a) Permite apreciar y valorar a los estudiantes

b) Favorece que los docentes y estudiantes perciban el aprendizaje logrado

c) Permite formar a los estudiantes

d) Facilita la entrega de docencia de mejor calidad

\section{Características de los estudiantes}

a) Estudiosos, esforzados

b) Interés genuino y espontáneo

c) Valoración de los pacientes

d) Les agrada atender pacientes

e) Les gusta recibir feedback

f) Valoración del docente

g) Evalúan bien el módulo

\section{Propias de la interacción del grupo}

a) Grupo pequeño favorece la interacción/contacto docente-estudiante

b) Participación activa

c) Alta interacción docente-alumnos

d) Se protegen

e) Se entregan feedback entre sí

f) Liderazgos positivos

g) Variedad de estudiantes y grupos

Respecto a la estructura del módulo, se mencionan como los más relevantes el diseño centrado en las necesidades del estudiante, la organización y estructura estandarizada, los objetivos precisos que favorecen el desarrollo de habilidades clínicas generales, propias de la especialidad y transversales, todo esto en un ambiente grato, seguro y controlado. Las siguientes citas textuales ilustran lo anterior:

"Si el estudiante ya sabe se le deja que él lo haga, si está aprendiendo se le explica cómo debe hacerlo haciendo una demostración, depende como se van dando las cosas. O sea se va adaptando a la necesidad del alumno y del paciente", "todos tratan que se pasen las mismas cosas, que se destaque lo mismo, van a ser evaluados de la misma manera", "el (estudiante) de marzo, va a aprender lo mismo que el de abril o el de diciembre, y va aprender lo mismo con el doctor $X, Y$ o Z". 
Tabla 5. Condiciones que favorecen la docencia en el módulo ambulatorio de enfermedades respiratorias del adulto dependientes del equipo docente

\section{Como individuos}

a) Habilidades docentes:

Enseñanza focalizada, seleccionada

Motivación

Saber dar feedback

Categorizar conocimientos

Estimular a la reflexión y autoevaluación

Estimular la participación

Estimular el autoaprendizaje

Orientación al cumplimiento de objetivos

Hace explícita las enseñanzas

Dar tareas y revisarlas

Ser ordenado

Fija reglas claras

Flexibilidad

Uso eficiente del tiempo

Uso intencionado de temas transversales

Enseñar/adecuar el lenguaje

Empatía con el estudiante y paciente

Valorar la interacción directa con los pacientes

b) Estilos y preferencias en los énfasis:

Asertivo, directo

Paternalista

Cercano con los alumnos

Participativo, aprendizaje autodirigido

Manejo de expectativas

Confianza en los alumnos

c) Modelar una buena relación médico-paciente

d) Adaptarse a las necesidades del estudiante

e) Adaptarse a las necesidades del paciente

f) Satisfacción personal con la actividad

g) Experiencia docente

h) Percibir y recibir valoración de la Institución

\section{Como grupo}

a) Interés común en la docencia

b) Alineados con los objetivos del curso

c) Hacen bien la tarea

d) Red de apoyo para que se realicen las otras actividades

e) Alto nivel de autonomía

f) No se quejan

g) Reuniones periódicas
Tabla 6. Condiciones mencionadas por los docentes que dificultan la docencia en el módulo ambulatorio de enfermedades respiratorias del adulto

\section{Recursos del módulo}

a) Dificultades en la captación de pacientes

b) La inasistencia de los pacientes es un problema frecuente y difícil de manejar

c) Faltan incentivos para estimular la participación de los pacientes

d) El recurso audiovisual es insuficiente y a veces falla

e) No se aprovecha el video como recurso docente

\section{Dependientes de los estudiantes}

a) No se hacen cargo de su aprendizaje (escaso interés por estudiar, se ausentan)

b) Poca concentración en la tarea

c) Alta dependencia de la supervisión

d) Escasa capacidad de darse feedback entre sí

e) Expectativas diferentes a los objetivos planteados

f) Pobre valoración del esfuerzo docente

g) Liderazgos negativos

\section{Dependientes de los docentes}

a) Alto costo académico-docente (compite con otros compromisos académicos)

b) Elevada demanda de tiempo y dedicación

c) Falta de adherencia a la metodología del módulo (uso de pautas, recursos, sistema de evaluación)

d) Falta tiempo para la reflexión sobre la práctica

e) Escaso trabajo en equipo (elevada autonomía e introversión, escaso feedback de los pares)

f) El uso del espejo requiere entrenamiento altamente específico (dificulta el control de los estudiantes, percepción de estar perdiendo el tiempo al otro lado del espejo)

De la docencia ambulatoria con atención de pacientes, los docentes mencionan como facilitadores, la posibilidad de modelar, de promover aprendizajes significativos, entregar feedback inmediato, reforzar los conocimientos teóricos, las múltiples oportunidades de aprendizaje y la integración de familiares en la atención del paciente. De la docencia ambulatoria en general, mencionan como facilitadores que los pacientes sean representativos de la realidad y que la super- 
visión directa sea constante. Esto lo expresan de la siguiente forma:

"Sirvo de modelo para la primera vez", "ser un modelo de cómo debiera ser la relación entre el paciente y el médico tratante", "pasar la materia a propósito del paciente... refuerzo de lo teórico a propósito del paciente... demostrando que lo teórico tiene aplicación".

\section{Facilitadores de la docencia dependientes de los estudiantes}

Los principales facilitadores de la docencia dependientes de los estudiantes mencionados fueron la posibilidad de valorarlos y de formarlos, el interés que ellos demuestran por el estudio, su agrado por atender a los pacientes, la fuerte interacción entre los estudiantes y el docente, su participación activa y los liderazgos positivos que se generan. Los docentes lo mencionan de la siguiente forma:

"La principal labor que se hace dentro de esta institución es formar a los estudiantes", "ellos están como ávidos de ser médicos", "es un cariño que le tienen a los pacientes, que es muy bueno", "hay grupos con los que tú te sientes cómodo, estimulado".

\section{Facilitadores de la docencia dependientes de los docentes}

Las condiciones que favorecen la docencia dependiente de los docentes pueden agruparse en variables individuales y grupales. Respecto a las variables individuales se mencionan habilidades docentes tales como motivar, saber entregar feedback, y categorizar los conocimientos; estilos asertivos, directos y la satisfacción personal con la actividad docente. Las frases siguientes ilustran lo anterior:

"Uno los estimula con preguntas, con los pacientes, y ellos estudian más y entonces uno ve caras contentas y se produce esto que el aprendizaje empieza así como una bola de nieve", "si sabes algo, tienes que expresarlo. O sea acá nadie te va a adivinar si tu sabes o no sabes si no lo cuentas", "yo les digo: mira son seis preguntas que al menos tienes que preguntarle", "la docencia me da las satisfacciones por las cuales estoy dispuesto a pagar".

Los condicionantes derivados de los docentes como grupo incluyen el interés común en la docencia, el alineamiento con los objetivos del curso y la red de apoyo entre los docentes que participan en esta actividad. Estas opiniones se expresan de la siguiente manera:
"Tenemos un interés común en la docencia, una cultura de la docencia, de querer mejorar...", "todos los docentes son más o menos homogéneos, todos los docentes se preocupan de cumplir los objetivos", "el tutor que está en docencia no hay que tocarlo, hay que dejarlo que esté allá, interrumpirlo lo menos posible...".

\section{Condiciones que dificultan la docencia en el módulo ambulatorio}

Los principales obstáculos para la docencia según la opinión de los docentes entrevistados, son aquellos dependientes de los recursos del módulo, especialmente la disponibilidad de pacientes y su ausencia a las citaciones; no menos importante es la falta de incentivos para los pacientes y las dificultades con el recurso audiovisual. Esto es expresado de la siguiente forma:

"A uno de repente lo complica, claro, porque ya no es tan estructurado como uno quisiera y uno tiene que en ese momento rellenar, y rellenar con cosas que sean adecuadas", "es un televisor con un monitor muy pequeño en blanco y negro...", "No ocupamos, por ejemplo, el sistema de video porque la cámara te graba muy a lo lejos casi una silueta", "el computador es muy viejo...".

Otros factores mencionados son los dependientes de los estudiantes, quienes no siempre se hacen cargo de su aprendizaje, se ausentan o demuestran poco interés por estudiar. Al respecto los docentes expresan lo siguiente:

"Hay estudiantes que se ausentan, no se interesan por aprender", "les gusta mucho el feedback, les gusta recibir feedback, pero no son muy buenos para dar feedback".

Entre los factores mencionados que no favorecen la docencia y que dependen de los propios docentes, destacan el costo académico que implica tener a un docente dedicado exclusivamente a esta actividad. Esto se refleja en las siguientes citas:

"Las tareas habituales que uno tiene, que se van acumulando... y una sobrecarga en la tarde para resolver cosas que no se pueden postergar", "el consumo de tiempo, yo te diría es lo primero, lo segundo y lo tercero".

\section{Evaluación de los estudiantes}

En la Tabla 7 se describe la evaluación del módulo ambulatorio efectuada por los estudiantes en el período 2004-2010 (596 encuestas), no encontrándose diferencias estadísticamente 
significativas en los diferentes años examinados. Los estudiantes declaran dedicar entre 6 y 9 horas/ semana al estudio personal, el logro de los objetivos de aprendizaje es superior al $85 \%$ y todas las dimensiones evaluadas tienen nota superior a 6,0.
La evaluación de la calidad de la docencia tutorial, medida por una segunda encuesta, se resume en la Tabla 8 (588 encuestas en total); es igualmente satisfactoria, no observándose diferencias estadísticamente significativas entre los distintos tutores

Tabla 7. Evaluación del módulo ambulatorio de enfermedades respiratorias del adulto efectuada por los estudiantes

\begin{tabular}{|c|c|c|c|c|c|c|c|}
\hline & & & Año & la eva & ión & & \\
\hline & 2004 & 2005 & 2006 & 2007 & 2008 & 2009 & 2010 \\
\hline $\mathrm{N}^{\circ}$ de encuestas & 93 & 105 & 87 & 71 & 86 & 76 & 78 \\
\hline Tiempo dedicado (horas) & 9,0 & 6,8 & 7,8 & 7,1 & 8,2 & 6,6 & 5,9 \\
\hline Objetivos (\% de logro) & 86,8 & 89,1 & 89,5 & 90,9 & 87,8 & 89,8 & 87,7 \\
\hline Dimensiones (Escala de 1,0 a & & & & & & & \\
\hline Métodos & 6,6 & 6,6 & 6,6 & 6,7 & 6,5 & 6,6 & 6,5 \\
\hline Profesores & 6,8 & 6,8 & 6,8 & 6,8 & 6,6 & 6,6 & 6,8 \\
\hline Fuentes de información & 6,6 & 6,7 & 6,5 & 6,8 & 6,6 & 6,7 & 6,7 \\
\hline Feedback & 6,1 & 6,4 & 6,4 & 6,7 & 6,6 & 6,6 & 6,6 \\
\hline Evaluación & 6,6 & 6,7 & 6,4 & 6,7 & 6,6 & 6,5 & 6,6 \\
\hline Organización & 6,8 & 6,8 & 6,8 & 6,7 & 6,6 & 6,6 & 6,7 \\
\hline Infraestructura y materiales & 6,8 & 6,9 & 6,9 & 6,9 & 6,8 & 6,8 & 6,8 \\
\hline Nota global & 6,5 & 6,7 & 6,6 & 6,8 & 6,5 & 6,6 & 6,6 \\
\hline Promedio & 6,6 & 6,7 & 6,7 & 6,8 & 6,6 & 6,7 & 6,7 \\
\hline
\end{tabular}

Los valores se expresan como valores promedio. No hubo diferencias significativas en los diferentes ítems de la encuesta en el período 2004-2010.

Tabla 8. Evaluación de la calidad de la docencia de los diez docentes que participaron en el módulo ambulatorio de enfermedades respiratorias del adulto efectuada por los estudiantes

\begin{tabular}{|lccccccc|}
\hline & \multicolumn{9}{c}{ Año de la evaluación } & & \\
& $\mathbf{2 0 0 4}$ & $\mathbf{2 0 0 5}$ & $\mathbf{2 0 0 6}$ & $\mathbf{2 0 0 7}$ & $\mathbf{2 0 0 8}$ & $\mathbf{2 0 0 9}$ & $\mathbf{2 0 1 0}$ \\
\hline N ${ }^{\circ}$ de encuestas & 109 & 101 & 82 & 66 & 77 & 68 & 85 \\
Dimensiones (Escala de 1,0 a 4,0) & & & & & & & \\
Docencia basada en pacientes & 3,6 & 3,7 & 3,6 & 3,7 & 3,6 & 3,7 & 3,8 \\
\hline Comunicación de los objetivos & 3,7 & 3,8 & 3,8 & 3,9 & 3,8 & 3,8 & 3,8 \\
\hline Evaluación & 3,3 & 3,6 & 3,6 & 3,6 & 3,5 & 3,7 & 3,7 \\
\hline Promoción de la comprensión & 3,8 & 3,9 & 3,9 & 3,9 & 3,8 & 3,8 & 3,8 \\
\hline Promoción del autoaprendizaje & 3,5 & 3,7 & 3,6 & 3,7 & 3,5 & 3,6 & 3,7 \\
\hline Control de la sesión & 3,6 & 3,8 & 3,6 & 3,8 & 3,7 & 3,6 & 3,7 \\
\hline Feedback & 3,3 & 3,7 & 3,7 & 3,7 & 3,6 & 3,7 & 3,7 \\
\hline Clima de aprendizaje & 3,8 & 3,9 & 3,9 & 3,9 & 3,8 & 3,8 & 3,9 \\
\hline Evaluación global & 3,8 & 3,9 & 3,9 & 3,9 & 3,8 & 3,7 & 3,9 \\
\hline
\end{tabular}

Los valores se expresan como valores promedio. No hubo diferencias significativas en los diferentes ítems de la encuesta en el período 2004-2010. 
Módulo de docencia ambulatoria de enfermedades respiratorias - I. Leiva et al

evaluados (datos no mostrados) ni entre los distintos años examinados, en cada uno de los ítems y en la evaluación global.

\section{Discusión}

En este estudio se ha evaluado, empleando metodologías cuantitativas y cualitativas, la calidad de la docencia ambulatoria de enfermedades respiratorias de cuarto año de medicina de la Escuela de Medicina de la Pontificia Universidad Católica de Chile, la cual ha sido evaluada favorablemente por los docentes y estudiantes, tendencia que, en el caso de los estudiantes, se mantiene en el período 2004-2010.

En la evaluación de la docencia, usualmente se mide la percepción de los estudiantes empleando cuestionarios específicos ${ }^{23}$, esta información es valiosa pero con sesgos naturales, por lo que se aconseja confrontarla con otras mediciones, como la observación y opinión de los propios docentes ${ }^{23,26,27}$. Hasta la fecha, en nuestra Escuela de Medicina, no se había realizado una evaluación sistemática de las percepciones de los docentes respecto a la docencia ambulatoria impartida. El empleo de la metodología cualitativa permitió contrastar la información obtenida de los estudiantes con la opinión de los docentes, identificando aspectos que permitirían mejorar la docencia y/o el grado de satisfacción de los estudiantes y docentes.

El cambio de la atención de los pacientes desde el ambiente hospitalario hacia el entorno ambulatorio, nos ha obligado a aprovechar las oportunidades de aprendizaje que ofrece este recurso ${ }^{1-5}$. Como se menciona en la literatura ${ }^{10}$, no es suficiente que los estudiantes observen como se realiza la atención ambulatoria o participen de ella, sino que se recomienda planificar estrategias educacionales específicas con el propósito de maximizar el uso de los recursos y las oportunidades de aprendizaje. Para ello, se recomienda que los grupos de estudiantes no sean muy numerosos; que los profesores tengan una formación docente básica e interés por la docencia clínica tutorial; disponibilidad de recursos incluyendo presupuesto y espacio físico especialmente habilitado; revisiones periódicas de los programas y evaluación del grado de satisfacción y logro de los objetivos. El cumplimiento de estos requisitos en el módulo ambulatorio de enfermedades respiratorias explicaría la evaluación favorable obtenida de los estudiantes y docentes.
Respecto a las limitaciones del estudio, no fue posible comparar nuestra experiencia con otros módulos de docencia ambulatoria, ya que no disponemos de estudios similares en el medio nacional; no se evaluó la percepción de los pacientes reales y simulados que participan en esta actividad, por lo cual no disponemos de información sistemática de su grado de satisfacción, sólo contamos con reportes anecdóticos de sus comentarios y del grado de adherencia a esta actividad (información no mostrada).

La información derivada de este estudio sugiere que la apreciación de los docentes es complementaria a la opinión de los estudiantes y permitió identificar aspectos que facilitan y dificultan la docencia ambulatoria de enfermedades respiratorias en nuestra Escuela de Medicina, información útil para la planificación de los cursos de pregrado de medicina, aunque no generalizable a otros programas o instituciones de educación superior de nuestro país.

Agradecimientos: Los autores agradecen a la Ps. Denisse Zúñiga y Ps. Gricelda Gómez por realizar las entrevistas y a los Drs. Rodrigo Moreno, Julio Pertuzé, María Teresa Beroíza y Jorge Jorquera, docentes del Departamento de Enfermedades Respiratorias.

\section{Referencias}

1. Barzansky B, Jonas HS, Etzel SI. Educational programs in US medical schools 1998-1999. JAMA 1999; 282: 840-6.

2. Dent JA, Angell-Preece HM, Ball HM, Ker JS. Using the Ambulatory Care Teaching Centre to develop opportunities for integrated learning. Med Teach 2001; 23: 171-5.

3. Cardarelli R, Sanders M. Ambulatory teaching and evidence-based medicine: applying classroom knowledge to clinical practice. Fam Med 2005; 37: 87-9.

4. Irby DM. Teaching and learning in ambulatory care settings: a thematic review of the literature. Acad Med 1995; 70: 898-31.

5. Levinsky NG. A survey of changes in the proportions of ambulatory training in internal medicine clerkships and residencies from 1986-87 to 1996-97. Acad Med 1998; 73: 1114-5.

6. Feltovich J, Mast TA, Soler NG. Teaching medical students in ambulatory settings in departments of internal medicine. Acad Med 1989; 64: 36-41. 
7. Fincher RM, Case SM, Ripkey DR, Swanson DB. Comparison of ambulatory knowledge of third-year students who learned in ambulatory settings with that of students who learned in inpatient settings. Acad Med 1997; 72 (10 Supppl 1): S130-2.

8. Declaration of Alma-Ata International Conference on Primary Health Care, Alma-Ata, USSR, 6-12 September 1978.

9. Canadian Institute for Health Information. National ambulatory care reporting system project report. Ottawa: CIHI, 1998.

10. Dent J. AMEE Guide № 26: clinical teaching in ambulatory care settings: making the most of learning opportunities with outpatients. Med Teach 2005; 27: 302-15.

11. Spencer J. Learning and teaching in the clinical environment. BMJ 2003; 326: 591-4.

12. Schuwirth LW, van der Vleuten CP. Challenges for educationalists. BMJ 2006; 333: 544-6.

13. Sprake C, Cantillon P, Metcalf J, Spencer J. Teaching in an ambulatory care setting. BMJ 2008; 337: 690-2.

14. Woolliscroft JO, Schwenk TL. Teaching and learning in the ambulatory setting. Acad Med 1989; 64: 644-8.

15. Rosso P, Velasco N, Moreno R. Reforma del curriculum de pregrado en la Escuela de Medicina de la Pontificia Universidad Católica de Chile: Objetivos, metodología y estado de avance. Rev Med Chile 1997; 125: 796-807.

16. Moreno R, Velasco N. Cambios curriculares en la Escuela de Medicina de la Pontificia Universidad Católica de Chile. Rev Chil Cir 1994; 46: 333-6.

17. Sánchez I, Riquelme A, Moreno R, Mena B, Dagnino J, Grebe G. Revitalizing medical education: the school of medicine at the Pontificia Universidad Católica de Chile. Clin Teach 2008; 5: 57-61.
18. Global Initiative for Chronic Obstructive Lung Disease (GOLD). Global Strategy for the diagnosis, management and prevention of chronic obstructive pulmonary disease 2009. www.goldcopd.com

19. Global Initiative for Asthma (GINA). Global Strategy for Asthma Management and Prevention. Updated 2010. www.ginasthma.org

20. Encuesta Nacional de Salud. ENS 2009-2010. Consumo de tabaco. Ministerio de Salud. Gobierno de Chile. www. redsalud.gov.cl

21. Charmaz K. Constructing grounded theory: a practical guide through qualitative analysis. Thousand Oaks, CA. Sage publications, 2006.

22. Patton MQ. Qualitative evaluation and research methods. Second edition, Newbury Park, CA: Sage Publications, Inc; 1990.

23. Bitran M, Mena B, Riquelme A, Padilla O, Sánchez I, Moreno R. Desarrollo y validación de un instrumento en Español para evaluar el desempeño de docentes clínicos a través de las percepciones de sus estudiantes. Rev Med Chile 2010; 138: 685-93.

24. Fincher RM, Albritton TA. The ambulatory experience for junior medical students at the Medical College of Georgia. Teach Learn Med 1993; 5: 210-3.

25. Seabrook MA, Lawson M, Baskerville PA. Teaching and learning in day surgery units: a UK survey. Med Educ 1997; 31: 105-8.

26. Sutkin G, Wagner E, Harris I, Schiffer R. What makes a good clinical teacher in medicine? A review of the literature. Acad Med 2008; 83: 452-66.

27. Irby DM, Gillmore GM, Ramsey PG. Factors affecting ratings of clinical teachers by medical students and residents. J Med Educ 1987; 62: 1-7. 